\title{
To evaluate Lipid Profiles in Patients with Chronic Kidney Disease in RajaRajeswari Medical College and Hospital, Bengaluru, Karnataka, India
}

\author{
${ }^{1}$ Sharanappa Patil, ${ }^{2}$ V Ajith Kumar, ${ }^{3}$ Sandhya Subramani
}

\begin{abstract}
Background and objectives: Dyslipidemia is a fairly common occurrence in chronic renal failure (CRF) patients. Cardiovascular mortality in patients with CRF is related to dyslipidemia. This study was done to identify the lipid abnormalities and its significance in CRF patients by comparing with age, sex, and body mass index (BMI) of matched healthy control population.
\end{abstract}

Materials and methods: In this study, 56 cases of CRF were taken and three fasting lipid profiles were estimated. Twentyfive age-, sex-, and BMI-matched healthy population were taken as controls. The data were entered in a master sheet and analyzed statistically.

Results: Dyslipidemia is seen in CRF patients. Even though the total cholesterol was high in CRF cases compared with controls, the change was not significant statistically. Triglycerides showed a statistically significant increase in CRF cases. The low-density lipoprotein cholesterol (LDL-C) was elevated in CRF cases but the change was not significant statistically. The high-density lipoprotein cholesterol (HDL-C), on the contrary, showed a statistically significant drop compared with controls.

Interpretation and results: Lipid abnormalities are common in CRF. Total cholesterol changes are not statistically significant. Triglycerides shows statistically significant increase in CRF cases when compared with normal. The LDL-C is increased in CRF patients, but it is not statistically significant when compared with controls. The HDL-C shows a statistically significant decrease in CRF patients compared with controls. These lipid abnormalities may be an important contributing factor to the cardiovascular mortality in patients with CRF.

Keywords: Chronic kidney disease, Dyslipidemia, Lipid profile.

How to cite this article: Patil S, Kumar VA, Subramani S. To evaluate Lipid Profiles in Patients with Chronic Kidney Disease in RajaRajeswari Medical College and Hospital, Bengaluru, Karnataka, India. J Med Sci 2018;4(2):31-34.

Source of support: Nil

Conflict of interest: None

\footnotetext{
${ }^{1}$ Senior Resident, ${ }^{2}$ Professor, ${ }^{3}$ Postgraduate Student

${ }^{1-3}$ Department of General Medicine, RajaRajeshwari Medical College \& Hospital, Bengaluru, Karnataka, India

Corresponding Author: V Ajith Kumar, Professor, Department of General Medicine, RajaRajeshwari Medical College \& Hospital, Bengaluru, Karnataka, India, Phone: +919845243763 e-mail: Ajithvenoor@yahoo.co.in
}

\section{INTRODUCTION}

Chronic kidney disease (CKD) results when a disease process affects the structural or functional integrity of the kidneys. Chronic kidney failure is the result of CKD.

Chronic renal disease features various abnormalities of lipid metabolism, which results in an exceedingly atherogenic profile. Although most striking lipid abnormalities are seen in nephrotic syndrome, hyperlipidemia characterizes renal disease of every cause.

Lipid abnormalities in CRF are very important, because atherosclerotic heart disease is the foremost cause of morbidity and mortality in patients with endstage renal disease (ESRD).

Cardiovascular diseases are the leading causes of death in ESRD largely as the result of progressively increasing age of ESRD patients and the broad constellation of uremia-associated features. When the kidney function has deteriorated and is no longer adequate to sustain life, renal replacement therapy, dialysis, or transplantation becomes necessary to maintain life. Hence, it is important to prevent the development of chronic renal insufficiency and subsequent progression to ESRD. ${ }^{1-4}$

Unfortunately, kidney disease in its early stages is generally asymptomatic. Early identification of patients at risk for CKD is essential. Major risk factors for the development and progression of CKD include type I diabetes, high blood pressure, protein barrier, family history of kidney disease, and increasing age.

The progression of kidney disease to end stage can be slowed by glycemic control (in diabetes), blood pressure control for the patients with high blood pressure and use of angiotensin-converting enzyme inhibitors. ${ }^{2-4}$

Cardiovascular disease is the major cause of death among patients with CRF and ESRD. In addition to impairing the microcirculation, hypertension may contribute to the development of atherosclerotic coronary artery disease particularly in the presence of many lipid abnormalities observed in ESRD.

The patients have reduced HDL-C and increased plasma triglyceride concentrations and there is defect in the cholesterol transport. Other factors that may contribute to atherosclerotic coronary artery disease in ESRD are reduced HDL-C synthesis and reduced activity of the reverse cholesterol pathway. 
The spectrum of dyslipidemia in patients with CKD and dialysis patients is distinct from that of the general population. It involves all lipoprotein classes and shows considerable variations depending on the stage of CKD. ${ }^{5-9}$

Also, a growing amount of clinical experience data suggests that lipids may be important in the development and progression of chronic renal disease. Potentially injurious lipid abnormalities are invariably present in these patients more likely to progress to ESRD.

So, the analysis of lipoprotein subclass in CRF patients is very much essential to assess the clinical outcome.

\section{MATERIALS AND METHODS}

\section{Source of Data}

Patients with CKD presenting to RajaRajeswari Medical College \& Hospital (RRMCH), Bengaluru.

\section{Data Collection}

Study subjects: The present study is a cross-sectional study conducted on 50 patients who are diagnosed with $\mathrm{CKD}$ and presenting to RRMCH during a period of 1 year.

\section{Inclusion Criteria}

- Patients with CKD

- Between age group of 18 and 80 years

- With known CKD irrespective of the etiology

- On conservative or dialysis treatment for CKD

- As evidenced radiologically (bilateral shrunken kidney/loss of corticomedullary differentiation) or biochemically (elevated blood urea, serum creatinine) for more than 3 months

\section{Exclusion Criteria}

- Patients with acute renal failure and nephrotic syndrome

- Patients on drugs affecting lipid metabolism like blockers, statins, steroids, and oral contraceptive pills

- Female patients who are pregnant

- Known hypothyroidism These patients were evaluated based on the proforma on the following guidelines:

- Clinical history and physical examination.

- Routine investigations like blood hemoglobin $(\mathrm{HB}) \%$, total count, differential count, blood sugar, and urine analysis.

- Renal parameters including blood urea, serum creatinine.

- Fasting lipid profile.

- Ultrasonogram abdomen.

All specimens were analyzed within 4 to 6 hours of collection. Total cholesterol and triglycerides in the plasma were measured enzymatically and then the cholesterol in the supernatant was measured after precipitation of apolipoprotein B (Apo-B) containing lipoprotein to determine the HDL-C. The LDL-C is estimated using Friedewald formula.

Friedewald formula appears to be the most practical and reliable method for determining LDL-C in clinical practice.

\section{LDL $-\mathrm{C}=$ Total cholesterol $-[\mathrm{HDL}-\mathrm{C}+($ Triglycerides/5) $]$}

Very-low-density lipoprotein (VLDL) is estimated by dividing the plasma triglycerides by 5 , reflecting the ratio of cholesterol to triglyceride in VLDL particles. This formula is reasonably accurate if the test resolution is obtained on fasting plasma and if the triglyceride level is less than $350 \mathrm{mg} / \mathrm{dL}$. The accurate determination of LDL-C level in conditions with triglyceride levels greater than this requires application of ultra centrifugation techniques (Beta quantification).

Consent: Informed consent was obtained.

\section{Statistical Tools}

The data collected regarding all the selected cases were recorded in a master chart. Data analysis was done with the help of computer using Epidemiological Information Package (EPI 2002). Using this software, range, frequencies, percentages, means, standard deviations, chi- square, and p-values were calculated. Kruskal-Wallis chi-square test was used to test the significance of difference between quantitative variables. A p-value less than 0.05 was taken to denote significant relationship.

\section{RESULTS}

The results are tabulated in Tables 1 to 4 and Graphs 1 and 2 .

Table 1: Age distribution

\begin{tabular}{llllll}
\hline & \multicolumn{2}{c}{ Cases } & & \multicolumn{2}{c}{ Control } \\
\cline { 2 - 3 } \cline { 5 - 6 } Age group (years) & No. & $\%$ & & No. & $\%$ \\
\hline $41-45$ & 1 & 1.8 & & 4 & 16 \\
$46-50$ & 29 & 51.8 & & 12 & 48 \\
$51-55$ & 19 & 33.9 & & 5 & 20 \\
$55-59$ & 7 & 12.5 & & 4 & 16 \\
Total & 56 & 100 & & 25 & 100 \\
Range & $45-57$ & & & $41-59$ & \\
Mean & 51.2 & & & 50.4 & \\
Standard deviation & 3.0 & & & 4.9 & \\
p-value & 0.1536 & & & \\
\hline
\end{tabular}

Not significant

Table 2: Sex distribution

\begin{tabular}{llllll}
\hline & \multicolumn{2}{c}{ Cases } & & \multicolumn{2}{c}{ Controls } \\
\cline { 2 - 3 } Sex & No. & $\%$ & & No. & $\%$ \\
\hline Male & 28 & 50 & & 10 & 40 \\
Female & 28 & 50 & & 15 & 60 \\
p-value & 0.7945 & & & \\
\hline
\end{tabular}

Not significant 
Table 3: Body mass index

\begin{tabular}{lll}
\hline BMI & Cases & Control cases \\
\hline Range & $22-26$ & $22-26$ \\
Mean & 24.55 & 24.76 \\
Standard deviation & 1.08 & 1.09 \\
p-value & 0.3668 & \\
\hline
\end{tabular}

Not significant

\section{DISCUSSION}

Chronic kidney disease results when a disease process affects the structural or functional integrity of the kidneys. Chronic kidney failure is the result of CKD. Cardiovascular disease is a major cause of mortality in patients with mild-to-moderate CKD and ESRD. Dyslipidemia has been established as a well-known traditional risk factor for cardiovascular disease in general population and it is well known that patient with CKD exhibits significant alterations in lipoprotein metabolism, which, in their most advanced form, may result in the development of severe dyslipidemia.

This study was done to identify the lipid abnormalities that occur in CRF patients admitted in RRMCH. A total of 56 cases who fulfilled the diagnostic criteria for CRF were included in the study. Twenty-five age-, sex-, and BMI-matched healthy controls who fulfilled the inclusion and exclusion criteria were taken for comparing the lipid profile.
Among 56 cases, the mean age was 51.2 years with the range of 45-57 years. The mean age of controls was 50.4 years and the range was $41-59$ years. There was no significant difference between cases and controls with regard to the age ( $p$-value 0.1536$)$. So, they can be compared.

There were equal number of males and females in the study group, 28 males and 28 females. Among the 25 controls, 10 were males and 15 were females. There was no significant difference between cases and controls as far as sex is concerned ( $p$-value 0.7945). The mean BMI of the cases was $24.55 \mathrm{~kg} / \mathrm{m}^{2}$.

The mean BMI of the controls was $24.76 \mathrm{~kg} / \mathrm{m}^{2}$. There was no significant difference between cases and controls with respect to BMI.

On analyzing the lipid profile and comparing the CRF cases with controls, we found that there is significant increase in triglycerides and significant decrease in HDL-C. The change in total cholesterol and LDL-C between cases and controls was not significant.

\section{Total Cholesterol}

The mean total cholesterol in the CRF cases was $213.6 \mathrm{mg} /$ $\mathrm{dL}$ and that of the controls was $207.8 \mathrm{mg} / \mathrm{dL}$. There was no statistically significant difference in this parameter ( $p$-value 0.1761). This observation was similar to the results obtained by Kimak et al in their work on plasma lipoproteins in CRF patients. They also concluded that total cholesterol is not increased significantly in patients with $\mathrm{CRF}^{10}$

Table 4: Lipid profile

\begin{tabular}{|c|c|c|c|c|c|c|c|}
\hline \multirow[b]{2}{*}{ Lipid } & \multicolumn{3}{|c|}{ Cases } & \multicolumn{3}{|c|}{ Controls } & \multirow[b]{2}{*}{$p$-value } \\
\hline & Range & Mean & $S D$ & Range & Mean & $S D$ & \\
\hline TC & 158-269 & 213.6 & 14.9 & $132-245$ & 207.8 & 22.1 & $0.1761 \mathrm{NS}$ \\
\hline TGL & $110-264$ & 205.9 & 44.9 & $112-180$ & 148.0 & 16.3 & $0.0001 \mathrm{~S}$ \\
\hline HDL & $16-75$ & 39 & 18.5 & $38-86$ & 60.7 & 14.3 & $0.0001 \mathrm{~S}$ \\
\hline LDL & $110-171$ & 139.4 & 16.4 & $110-172$ & 127.2 & 15.6 & $0.1031 \mathrm{NS}$ \\
\hline
\end{tabular}

SD: Standard deviation; TC: Total cholesterol; TGL: Triglyceride; NS: Not significant; S: Significant

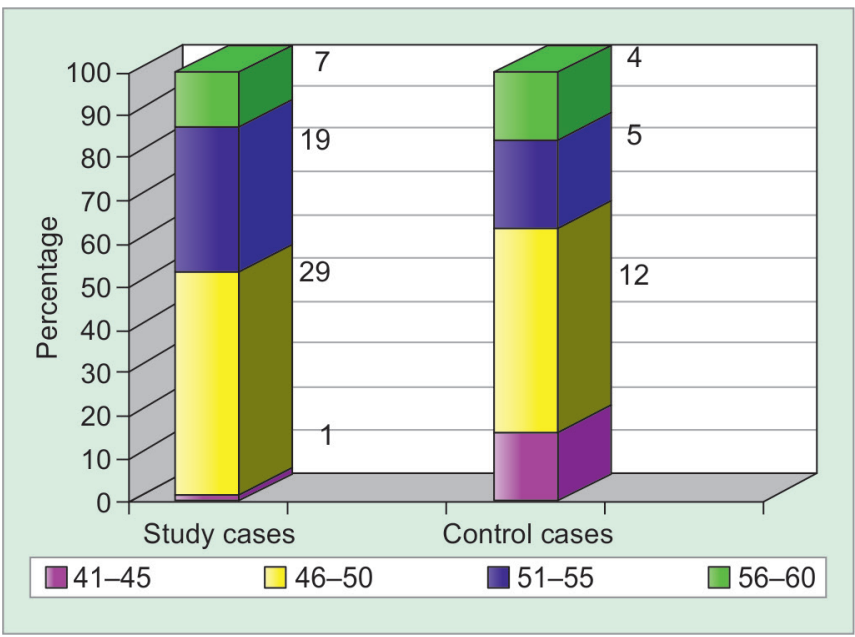

Graph 1: Age distribution

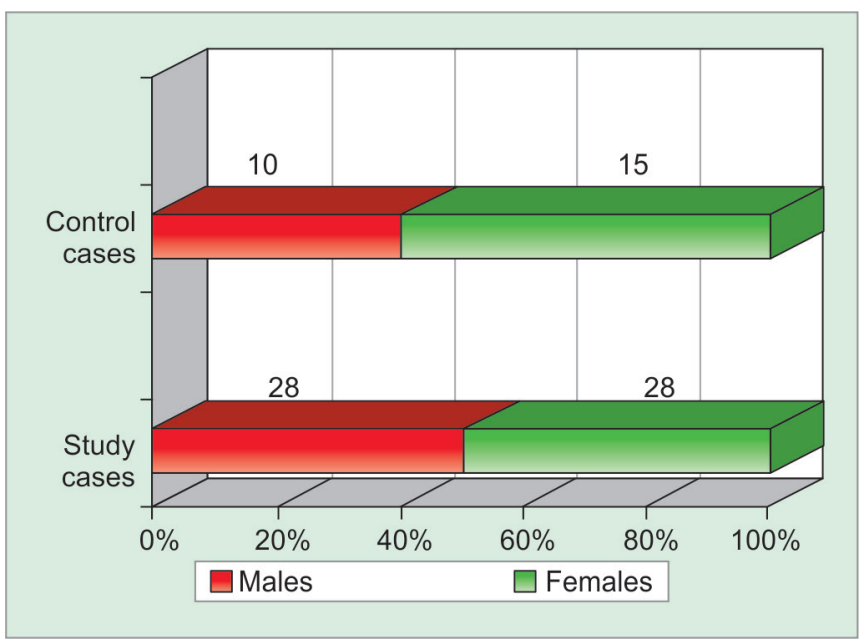

Graph 2: Sex distribution 


\section{Triglycerides}

Significant increase in serum triglycerides was seen in cases when compared with controls. Mean triglycerides was $205.9 \mathrm{mg}$ in cases and $148 \mathrm{mg} / \mathrm{dL}$ in controls ( $p$-value 0.0001). This result is in concordance with the work done by Kimak et al, in which they demonstrated significant increase in triglycerides, LDL, and Apo-B concentrations. ${ }^{11}$ In another study done by Bhagwat et al, ${ }^{11}$ they concluded that CRF patients were having marked triglyceridemia of $232 \mathrm{mg} / \mathrm{dL}$ as compared with controls (p-value $<0.01$ ). Another Indian study on dyslipidemia in patients with CRF and renal transplantation by Shah et $\mathrm{al}^{12}$ demonstrated that triglycerides level was elevated significantly in CRF patients on conservative management. These results show that hypertriglyceridemia is an important lipid abnormality in patients with CRF.

\section{Low-density Lipoprotein Cholesterol}

Our study demonstrated an increase in LDL-C between cases and controls (139.4 vs $127.2 \mathrm{mg} / \mathrm{dL}$ ). This was not significant statistically, p-value (0.1031). This was similar to the study by Bhagwat where they found that LDL-C in CRF patients showed an increase compared with controls, which is not statistically significant. Study by Kimak et al showed results not comparable to our study. The LDL-C showed significant increase among CRF patients compared with controls in their study. ${ }^{10}$ Although the total concentrations of LDL are not significantly increased, there is predominance of small dense particles which are particularly susceptible to oxidation in CRF. These small particles are thought to be more atherogenic than larger LDL substrates. ${ }^{13}$

\section{High-density Lipoprotein Cholesterol}

Our study demonstrated a significant decrease in HDL in CRF cases when compared with controls (39 vs 60.7 $\mathrm{mg} / \mathrm{dL}$, p-value 0.001). This was in concordance with the results obtained by Bhagwat et al where they found HDL-C to be significantly low $(20 \pm 11) \mathrm{mg} / \mathrm{dL}$ (p-value less than 0.001) in CRF groups. ${ }^{11}$ Patients with CKD generally have reduced plasma HDL-C concentrations when compared with nonuremic individuals.

\section{CONCLUSION}

Lipid abnormalities are common in CRF. Total cholesterol changes are not statistically significant. Triglycerides show statistically significant increase in CRF cases when compared with normal. The LDL-C is increased in CRF patients, but it is not statistically significant when compared with controls. The HDL-C shows a statistically significant decrease in CRF patients compared with controls.

\section{REFERENCES}

1. Dietary lipid metabolism and chronic renal failure. In: Davidson's principles \& practice of medicine. 20th ed. pp. 444, 445, 485-496.

2. Chronic kidney disease: Guidelines for GFR estimation. In: API text book of medicine. 8th ed. Vol. 1. p. 735.

3. Lipid abnormalities in chronic kidney disease. In: Harrisons principles of internal medicine. 17th ed. Vol. 2. p. 1763.

4. Lipid abnormality in CKD. In: Current medical diagnosis \& treatment; 2008. pp. 793-800.

5. Lipid abnormalities in chronic kidney disease. In: Oxford textbook of clinical nephrology. 2nd ed. Vol. 3. p. 1840.

6. Lipid abnormalities in chronic kidney disease progression In: Brenner \& Rector's, The Kidney, 7th ed. Vol. 2. p. 1975.

7. Moorhead JF, Chan MK, El-Nahas M, Varghese Z. Lipid nephrotoxicity in chronic progressive disease. Lancet 1982 Dec 11;2(8311):1309-1311.

8. Myhre E, Gjone E, Flatmark A, Hovig T. Renal failure in familial lecithine acetyltransferase deficiency. Nephron 1997;8:840-852.

9. Rigatto C, Parfrey P, Foley R, Negrijn C, Tribula C, Jeffery J. Congestive heart failure in renal transplant recipients, risk factors, outcomes, and relationship with ischemic heart diseases. J Am Soc Nephrol 2002;13:1084-1090.

10. Kimak E, Solski J, Janicka L, Duma D, Zagojska M. Plasmalipoproteins in patients with CRF. Int Urol Nephrol 1997;29(5):597-601.

11. Bhagawat R, Joshi SP, Salgia P, Sepha A. Lipid abnormality in chronic renal failure. Indian J Clin Biochem 1997 Jan;12(1):81-85.

12. Shah B, Nair S, Sirsat RA, Ashavaid TF, Nair KG. Dyslipidemia in patients with CRF and renal transplantation. J Postgrad Med 1994 Apr-Jun;40(2):57-60.

13. Rajman J, Harpel L, McPake D, et al. Low density lipoprotein profile in CRF. Nephrol Dial Transplant 1998;13:2281. 